Research Article

\title{
Knowledge, Attitudes, and Management of General Practitioners of the Hospital Districts of Ouagadougou about Migraine (Burkina Faso)
}

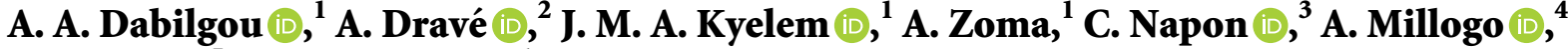 \\ K. Karfo iD, $\mathbf{5}$ and J. Kaboré (iD ${ }^{1}$ \\ ${ }^{1}$ Department of Neurology, University Hospital Yalgado Ouedraogo, Ouagadougou, Burkina Faso \\ ${ }^{2}$ Department of Neurology, Regional University Hospital of Ouahigouya, Ouahigouya, Burkina Faso \\ ${ }^{3}$ Department of Neurology, University Hospital of Bogodogo, Ouagadougou, Burkina Faso \\ ${ }^{4}$ Department of Neurology, University Hospital of Sourou Sano, Bobo Dioulasso, Burkina Faso \\ ${ }^{5}$ Department of Psychiatry, University Hospital Yalgado Ouedraogo, Ouagadougou, Burkina Faso
}

Correspondence should be addressed to A. A. Dabilgou; dabilgouanselm@yahoo.fr

Received 26 July 2021; Revised 14 October 2021; Accepted 2 November 2021; Published 18 November 2021

Academic Editor: Filippo Brighina

Copyright $\left({ }^{\circ} 2021\right.$ A. A. Dabilgou et al. This is an open access article distributed under the Creative Commons Attribution License, which permits unrestricted use, distribution, and reproduction in any medium, provided the original work is properly cited.

Background. Migraine is a common neurological disorder characterized by severe headache attacks that may be debilitating. The objective of this study is to determine the knowledge and attitudes of general practitioners in the hospital districts of the city of Ouagadougou on migraine. Methods. This cross-sectional study was carried out in hospital districts of Ouagadougou. The data were collected during three months from February 1 to April 30, 2020. Results. The study included 116 general practitioners. Thirteen percent of them were suffering from migraine. All participants had previous experience with migraine diagnosis before the survey. Eighty percent of general practitioners had a good level of knowledge of ICDH-3 criteria (knowing 6-7 criteria). The most widely recognized IHS criteria were pulsatility quality (93.1\%), photophobia or sonophobia (80.2\%), and mild-to-moderate intensity $(80 \%)$. Ninety-five $(81.9 \%)$ general practitioners rarely ordered brain imaging. The most common acute treatments were nonsteroidal inflammatory drug $(39.47 \%)$, paracetamol $(44.74 \%)$, and derivate of ergot $(3.95 \%)$. The most common preventive treatments were amitriptyline $(27.8 \%)$, derivate of ergot $(18.9 \%)$, and NSAID (16.7\%). The majority of general practitioners (56.9\%) have referred headache patients to a colleague or specialist. Conclusions. Our study found that diagnostic criteria and acute treatment of migraine were well known by the majority of general practitioners, in contrast of preventive treatment.

\section{Background}

Migraine is a common neurological disorder characterized by severe headache attacks that may be debilitating $[1,2]$. The diagnosis is based on the International Classification of Headache Disorders (ICHD-3 beta version) diagnostic criteria published in 2013. Migraine is one of the most common conditions presenting to both a primary care and general neurology practice [3]. The World Health Organization has declared migraine a major public health problem due to a lack of knowledge about its causes and effective treatment options [4]. Primary care is an important setting for the management of migraine, and in many countries, most migraine consultations occur in this context [5]. Migraineurs who consult PCPs for migraine may not receive a correct diagnosis [6] and/or may not receive adequate treatment [7]. There have been few studies investigating how to improve migraine education in the primary care setting [8-10]. There are African recommendations on the management of migraine in adults [11]. To our knowledge, there are not yet studies on the evaluation on the migraine management of GPs in Sub-Saharan Africa, as in Burkina Faso. The objective of our study was to assess the knowledge of general practitioners of the five hospital districts of 
Ouagadougou in order to optimize their knowledge, attitudes, and practices in the face of migraine.

\section{Materials and Methods}

2.1. Study Profile. We carried out a descriptive cross-sectional study in the five hospital districts of the city of Ouagadougou, during a period of 3 months from February 1 to April 30, 2020.

2.2. Study Population. The study population consisted of GPs of the five hospital districts of Ouagadougou who consented to participate in the survey.

2.3. Sampling. This was a systematic nonrandom sample of general practitioners working in the five hospital districts of the city of Ouagadougou.

2.4. The Questionnaire. Data were collected using a selfadministered anonymous questionnaire comprising four parts: sociodemographic data of participants (age, sex, seniority, medical background, specific training on migraine, and internship in neurology); familiarity of migraine (number of migraineurs consulted per year and age of migraine patients at the time of diagnosis); migraine diagnosis (criteria of migraine, types of investigations in case of migraine, indication of these investigations, colleague opinion award, use of a headache diary, assessment of the handicap generated by migraine, and complications of migraine); and management of migraine (knowing VAS, type of acute treatment, conditions of initiation of acute treatment, conditions of initiation of prophylactic treatment, type of prophylactic treatment, and duration of prophylactic treatment). The questionnaire was semistructured in which the participant had to answer with $<$ yes, $<$ no, or $<$ do not know or to choose one or more answers among those which were proposed to him. Some questions were open-ended. The initial version of the questionnaire was composed by three of the authors of this work (ZA, DAA, and CN) and was changed to the final form after revision for content validity and clarity by a pretest study that was carried randomly in 15 GPs working in other medical centers.

\subsection{Data Collection and Analysis}

2.5.1. Procedure of Collection. GPs from the five district hospitals in the city of Ouagadougou were visited in the time of the survey. In each district, we first met with the district authorities to obtain lists of GPs and their consultation program. We met all the GPs and present them the authorization of Health Ministry of Burkina Faso. All the GPs presented in the time of the visit were asked to answer the questionnaires at their workplace. After receiving their verbal consent to participate, they received the paper version of the questionnaire. Depending on their availability, the questionnaire was completed in the time of the visit or later and returned back with care unit supervisors.
2.5.2. Data Analysis. The data collected were analyzed by using Epi Info version 7.2. The quantitative variables were expressed as means and standard deviation. The qualitative variables were expressed in numbers and in percentage.

2.6. Assessments. We questioned GPs on the criteria which in the face of a headache make them pose the diagnosis of migraine without aura. They were evaluated according to knowing ICDH 3 diagnosis criteria of migraine without migraine. The level of knowledge was assessed into 4 levels: low level of knowledge: 0-4 criteria; adequate level of knowledge: 5 criteria; good level of knowledge: 6-7 criteria; and very good level of knowledge: all ICDH-3 criteria. Management of migraine was evaluated according to international guidelines. For acute treatment, the correct response was accepted if it contained the following: NSAIDs and/or aspirin and/or aspirin and metoclopramide and/or paracetamol or triptans. For the prophylactic treatment, the valid response was amitriptyline and/or oxetorone and/or pizotifen and/or propranolol and/or topiramate, valproate de sodium, and carbamazepine.

\section{Results}

3.1. Sociodemographic and Professional Characteristics. Our study population included 116 GPs of the 5 health districts of the city of Ouagadougou. The mean age of participants was 32 years. The majority of them were men (54.3\%) and were graduated before 2015 (74.1\%). The professional experience of the participants was 3.69 years. Forty-six GPs (39.6\%) had already completed an internship in neurology during their initial medical training. All the GPs worked in public health centers. The socioprofessional data of GPs are given in Table 1.

3.2. Experience of Migraine. Sixteen participants declared themselves migraineur (13.8\%) with a majority of women (56.2\%). All participants had previous experience with migraine diagnosis before the survey. Of them, 95 (81.9\%) reported having consulting 5-35 migraineurs during last year. For $81 \%$ of GPs, the mean age of migraineurs was between 20 and 30 years.

3.3. Migraine Diagnosis. Table 2 shows the criteria used by the GPs to establish the diagnosis of migraine without aura $(N=116)$. Twenty-three GPs $(19.8 \%)$ had very low knowledge of ICDH-3 criteria of migraine without aura and 93 $(80.2 \%)$ had a good level of knowledge of ICDH-3 criteria. The most widely recognized IHS criteria were pulsatility quality (93.1\%), photophobia or sonophobia $(80.2 \%)$, and moderate or severe intensity (80\%). At least sixty-seven $(57.8 \%)$ GPs gave a wrong answer. The wrong responses were bilateral location (53.5\%), very severe form of headache $(19.8 \%)$, sudden onset $(6.9 \%)$, and headache in helmet (5.2\%). According to investigations in case of migraine, 95 (81.9\%) GPs rarely ordered brain imaging and 21 (18.1\%) regularly or systematically prescribed brain imaging. The 
TABLE 1: Sociodemographic and professional characteristics of GPs $(N=116)$.

\begin{tabular}{|c|c|c|}
\hline Variable & Study population $(N=116)$ & Percentage \\
\hline \multicolumn{3}{|l|}{ Age group (years) } \\
\hline$<30$ & 41 & 35.3 \\
\hline $30-40$ & 72 & 62.1 \\
\hline$>40$ & 2 & 2.6 \\
\hline Male gender & 63 & 54.3 \\
\hline History of migraine & 16 & 13.8 \\
\hline \multicolumn{3}{|l|}{ Professional experience (years) } \\
\hline $1-5$ & 86 & 74.1 \\
\hline $5-10$ & 25 & 21.6 \\
\hline$>10$ & 5 & 4.3 \\
\hline Completed internship in neurology & 46 & 39.6 \\
\hline Number of migraineurs received last year & 50 & 43.1 \\
\hline
\end{tabular}

TABLE 2: Criteria used to establish the diagnosis of migraine $(N=116)$.

\begin{tabular}{lcc}
\hline Diagnostic criteria for migraine & Study population $(N=116)$ & Percentage \\
\hline Pulsating quality & 108 & 93.1 \\
Moderate or severe form of headache & 93 & 80 \\
Presence of photophobia or phonophobia & 93 & 80.2 \\
Increase in headache with physical activity & 92 & 79.3 \\
Bilateral headache & 67 & 57.8 \\
Presence of nausea or vomiting & 62 \\
Pain lasting for about 4-72 hours & 60 \\
Unilateral headache & 47 & 53.4 \\
Severe or very severe form of headache & 23 & 51.7 \\
Sudden onset & 8 & 19.8 \\
Headache in helmet & 6 & 6.9 \\
\hline
\end{tabular}

patients who have benefited from the brain scan were those presenting an abnormal neurological examination (67.2\%), a migraine after 50 years $(63.79 \%)$, an atypical aura $(51.72 \%)$, and an attack duration over 4 hours (2.6\%) and over 7 days (18.10\%). The other investigations prescribed by GPs were an ophthalmologic exploration (56.03\%), an electroencephalogram (37.07\%), and a sinus X-ray (18.10\%).

3.4. Migraine Management. Sixty-nine participants $(59.48 \%)$ answered the question regarding visual analog scale for pain. Of these, $49(86.95 \%)$ gave a correct definition of visual analog scale for pain while 20 $(17.24 \%)$ reported using it in routine practice. The strategies of acute treatment were based on VAS for pain (90.5\%) and repercussion on daily life (97.4\%). Table 3 gives the drugs used by GPs in the management of acute and chronic migraine. Of 115 GPs who answered the question relative to medications of migraine acute treatment, $60(39.47 \%)$ said they would use an NSAID, 68 (44.74\%) paracetamol, and $3.95 \%$ derivate of ergot. The proportion of correct answers was $98 \%$. All the participants had answered the question concerning the initiation of prophylactic treatment of migraine. The majority of them thought that migraine prophylactic treatment should be started when the acute treatment has failed (66.4\%), according to the impact on daily life $(63.8 \%)$, frequency of attacks $(62.9 \%)$, when patient had more than
3-6 episodes of acute migraine per month (44.8\%), and intensity of headache on VAS for pain (25\%). A total of 90 (77.6\%) GPs answered the question according to prescription of prophylactic treatment. Twenty-five respondents (27.8\%) would use amitriptyline, 25 (18.9\%) would use derivate of ergot, and 15 (16.7\%) would use NSAID. Forty percent of respondents would use recommended molecule. Of the 114 respondents, 43 (37.7\%) GPs regularly assessed the effectiveness of the prophylactic treatment of migraine. This evaluation took place one month (55.8\%), 3 months (39.5\%), and 6 months after initiation of prophylactic treatment $(4.6 \%)$. One hundred and eleven GPs answered the question on the duration and the effectiveness of prophylactic treatment. Of these, 18 (16.2\%) judged effectiveness after reducing headache frequency by $25 \%, 69(62.2 \%)$ after $50 \%$ reduction in headache frequency, and 24 (21.6\%) after $75 \%$ reduction in headache frequency. When the prophylactic treatment was effective, that was stopped 3 months after its initiation (12.9\%), 6 months after (40.5\%), 12 months after $(6 \%)$, or never $(26.7 \%)$.

3.5. Referral Pattern. The majority of general practitioners (56.9\%) have referred headache patients to a colleague or specialist. The main reasons for referral were better treatment $(69.7 \%)$, systematically $(25.75 \%)$, and for diagnosis (4.54\%). The full version of study questionnaire is given in Table 4. 
TABle 3: Drugs used by GPs in the management of acute and chronic migraine.

\begin{tabular}{lcc}
\hline Variable & $\begin{array}{c}\text { Number } \\
(n=115)\end{array}$ & Percentage \\
\hline Drugs used in the management of migraine $(n=115)$ & \\
Paracetamol & 56 & 48.7 \\
NSAID & 46 & 40 \\
Caféine & 6 & 5.2 \\
Derivate of ergot & 5 & 4.3 \\
Triptan & 3 & 2.6 \\
Tramadol & 2 & 1.7 \\
Amitriptyline & 1 & 0.9 \\
Propranolol & 1 & 0.9 \\
Drugs used in the management of chronic migraine $(n=90)$ \\
Amitriptyline & 25 & 27.8 \\
Derivate of ergot & 17 & 18.9 \\
NSAID & 15 & 16.7 \\
Topiramate & 12 & 13.3 \\
Triptan & 11 & 12.2 \\
Propranolol & 8 & 8.9 \\
Ergotamine, caféine, and & 6 & 6.7 \\
paracetamol & 4 & 4.4 \\
Paracetamol & 2 & 2.2 \\
Tramadol & 2 & 2.2 \\
Paracetamol and codeine & 2 & 2.2 \\
Paracetamol, caféine, and & & \\
codeine & & \\
\hline
\end{tabular}

\section{Discussion}

This cross-sectional study is the first kind in Burkina Faso, a developing country in West Africa to determine the knowledge, attitudes, and practices of GPs working in the primary health level about migraine. Patients received the right diagnosis only just at the lowest level in primary health care services $[12,13]$. The majority of GPs in our study (80.2\%) had a good level of knowledge of ICDH criteria of migraine without aura. This high level of knowledge could be explained by the familiarity of GPs toward migraine. Most GPs had experienced migraine attacks (13.8\%), in high proportion than those in general population (5.6\%) [14]. Otherwise, healthcare professionals (HCPs) have stressful jobs, are frequently on rotating work shifts, undergo emotional stress, and work long hours every day because of their job requirements $[15,16]$. This high prevalence could be explained by the fact GPs with migraine may be more interested in the study than other doctors. Similar proportion was observed for primary health care physicians in Turkey (15\%) [17], but higher proportion for GPs was observed in Spain (27.4\%) [18] and in Germany (24.6\%) [19]. All the GPs in our study had already made a migraine diagnosis before the survey, in contrast with the study of Gültekin (8.3\%) [17]. This situation could be explained by the fact that a great number of them $(39.6 \%)$ had already completed an internship in neurology during their initial medical training. The vast majority of GPs $(81.9 \%)$ had already reported having received 5-35 migraineurs last year. This frequency seems lower compared to that encountered in other countries such as Pakistan, where among 449 family physicians, $15.1 \%$ of them had reported seeing $21-50$ migraine patients per month [20]. The most common diagnostic criteria knew by GPs were pulsatile quality (93.1\%), presence of photophobia or phonophobia $(80.2 \%)$, mild or severe form of headache $(80 \%)$, and increase in headache with physical activity (79.3\%). These results were different with the study of Gültekin which found unilateral headache (53\%), throbbing characteristic (47\%), presence of nausea or vomiting (45.5\%), and presence of photophobia or phonophobia (41.7\%) [17]. Regarding paraclinical explorations, studies have shown that primary care GPs have a great tendency to prescribe radiological investigations in the event of chronic headache [21, 22]. Regarding investigations, $81.9 \%$ of GPs prescribed brain scan on a case-by-case basis, in the event of abnormal clinical examination (67.2\%), migraine attacks appearing after the age of 50 years (63.8\%) and atypical aura (51.72\%). All these results were in line with revised French guidelines [23]. However, some requests were unjustified, namely, in cases of migraine attacks lasting 4 hours (18.1\%) and those dating more than 7 days (2.6\%). The brain scan was systematically requested in $18.1 \%$, despite the recommendations [23]. A study from Turkey found that $33.1 \%$ of PHCP had expressed the view that laboratory inquiry and brain screening are necessary for migraine diagnosis [17]. Other investigations such as an ophthalmologic exploration (56\%), an electroencephalogram (37.1\%), and a sinus X-ray (18.1\%) are not justified because there is no indication to perform these investigations in a patient with migraine defined according to IHS criteria (professional agreement) [23]. The most common acute treatments were paracetamol (44.7\%), NSAID (39.5\%), and derivate of ergot (4\%). In Pakistan, 50.9\% of physicians identified NSAIDs before triptans (34.6\%), as being the most common effective treatment for acute migraine [20]. According to MacGregor, a significant proportion of PCPs prescribe simple analgesics and nonsteroidal anti-inflammatory drugs (NSAIDs) for migraine (even for severe attacks), rather than migraine-specific treatments [7]. On the other hand, in industrialized countries such as France, more than $75 \%$ of GPs prescribed triptans as part of the treatment of migraine attacks [24]. Migraine-specific treatments, triptans $(2.6 \%)$ and ergotamine derivatives (4\%), were less prescribed by GPs in our context because of their unavailability and their high cost. According to prophylactic treatment, the majority of GPs thought that it should be started when the acute treatment has failed (66.4\%), worse impact on daily life (63.8\%), and increase of migraine attacks (62.9\%). These conditions correspond to the recommendations of the African guideline [11]. However, GPs in comparison with neurologists tend to institute prophylactic treatment for lower thresholds [25]. Twenty-two percent of GPs did not answer the question regarding the type of medications they can use for prophylactic treatment, for fear of giving wrong answer. Among the respondents, $39.8 \%$ of them had used recommended preventive medications, namely, amitriptyline (27.8\%), topiramate $(13.3 \%)$, and propranolol (8.9\%). Pharmacologic agents with the most clinical experience in chronic migraine (CM) include beta-blockers, topiramate, and amitriptyline [26-29]. In our series, two GPs prescribed molecules reserved for the prophylactic treatment in the treatment of migraine attacks, namely, propranolol and amitriptyline. This trend was also observed in Pakistan where 19\% of physicians prescribed topiramate in the treatment of migraine attacks [19]. Obviously, this was a therapeutic error that could worsen the 
TABLE 4: Full version of the questionnaire.

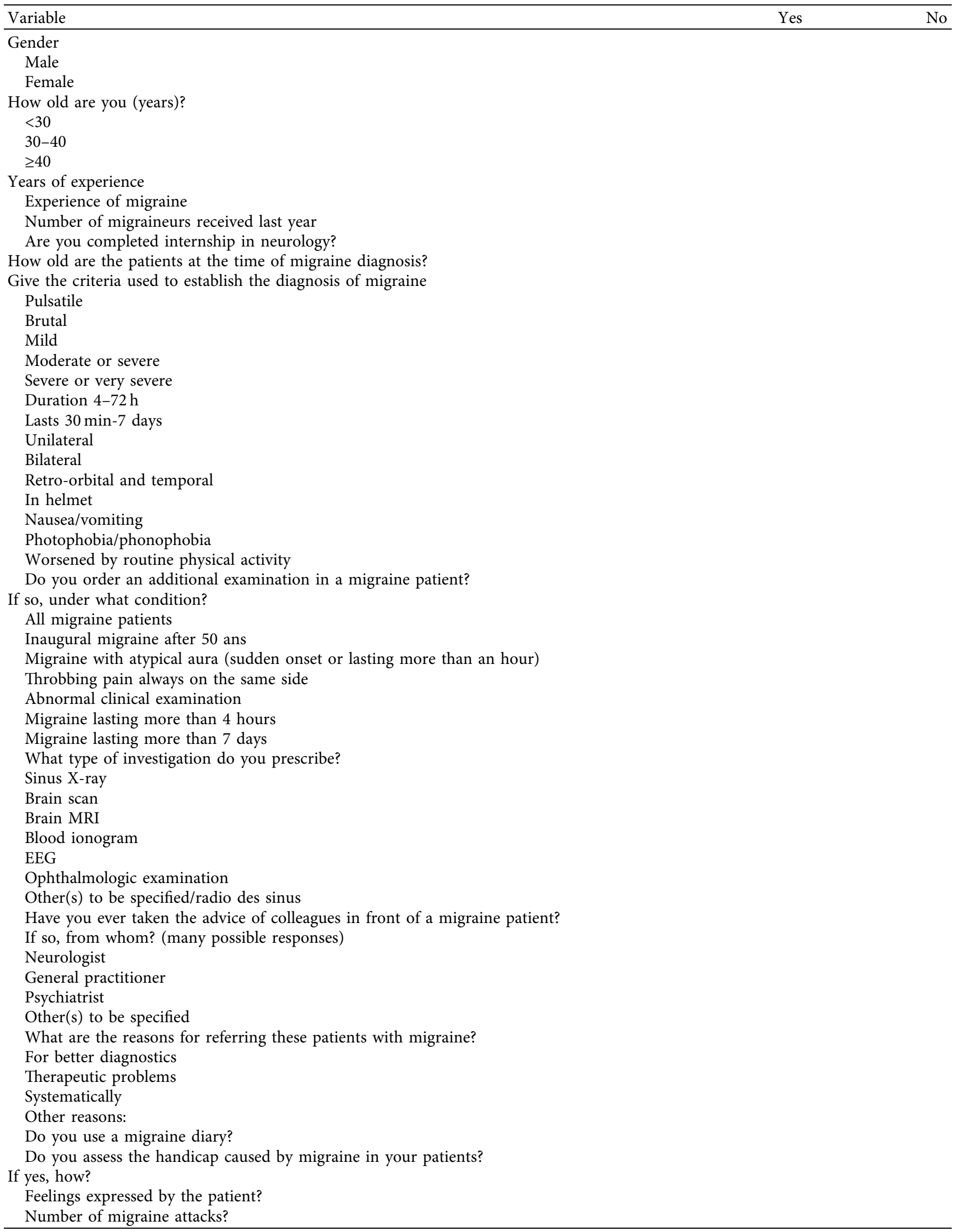


TABLE 4: Continued.

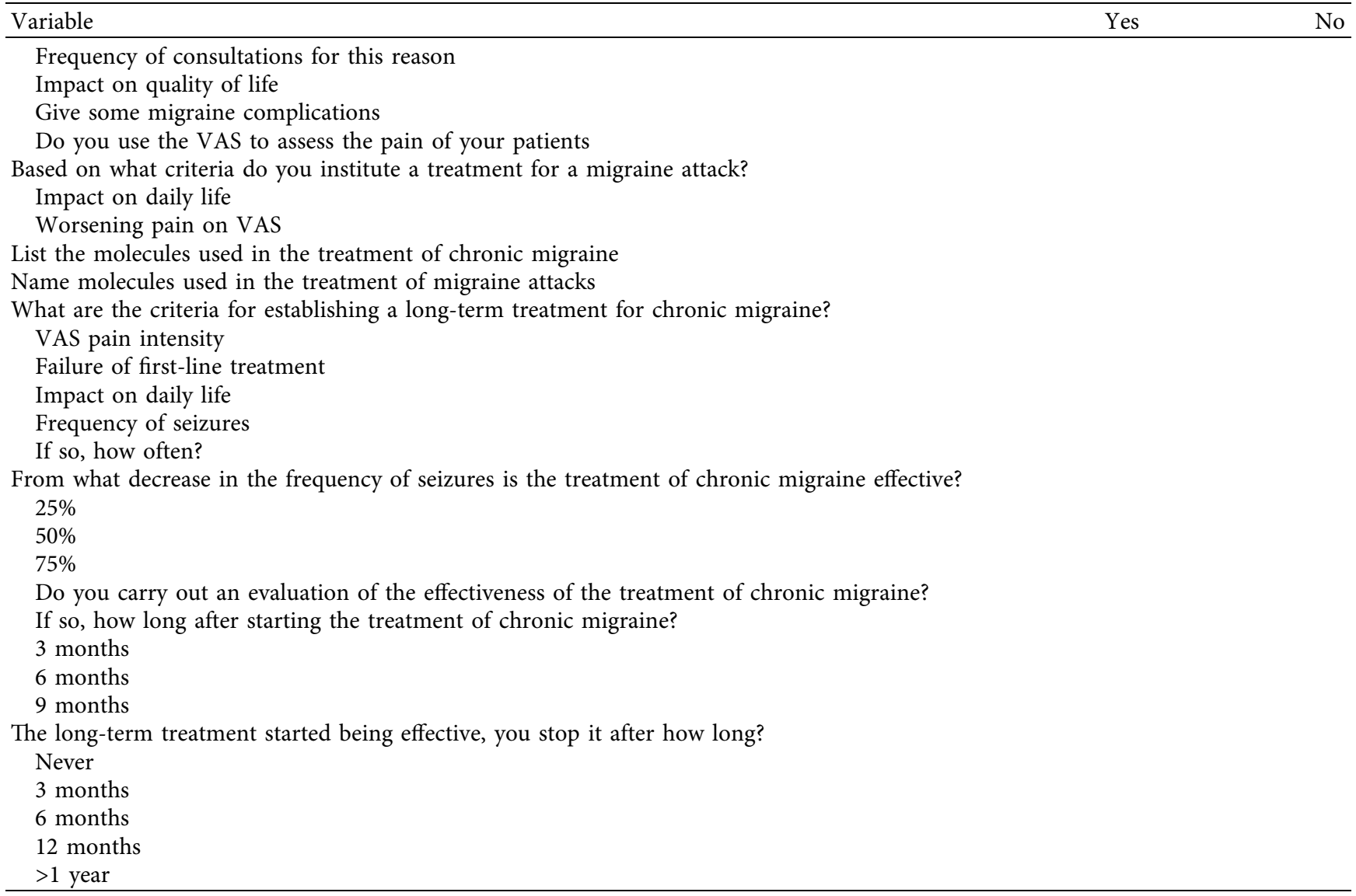

migraine attack or the need to train doctors on the issue. Conversely, several drugs for the treatment of the migraine attack were prescribed in the prophylactic treatment of migraine, namely, derivate of ergot (18.9\%), NSAID (16.7\%), tramadol (2.2\%), and paracetamol (4.4\%). Acute treatments are allowed for patients who are having frequent and severe migraine episodes [30]. Only 38\% of respondents regularly assessed the effectiveness of prophylactic treatment. This low percentage could be explained by the fact that most patients no longer consult after the improvement of the seizures. Thirtynine percent of respondents prescribed monitoring of the efficacy of the prophylactic treatment of migraine within the time limit (at 3 months), in accordance with African recommendations, while the majority of respondents (55.8\%) did so at one month early [12]. The majority of respondents (62.2\%) judged the effectiveness after a $50 \%$ reduction in the frequency of seizures, in accordance with the literature [31]. The majority of respondents stopped the prophylactic treatment of migraine before 8 months (53.4\%), 6\% after 12 months, and 26.7\% never stopped the prophylactic treatment of migraine. This attitude is at odds with the data in the literature which states that the discontinuation of the prophylactic treatment should be done after 8-12 months [31]. According to referral pattern, $56.9 \%$ of GPs would refer the patients to another GP or specialist. Of them, $69.7 \%$ would refer the patients for the treatment of the migraine, in higher proportion than in a study from India which states that $5 \%$ of general physicians were referring the patients to the neurologist for the treatment [32]. However, the most common reason for referral was management of migraine $(69.7 \%)$, in contrast with the study of Fearon in which diagnostic clarification (53.5\%) was the most common reason [33].

\section{Limitations of the Study}

This cross-sectional study had some limitations. Indeed, the anonymity of the data collection sheet did not allow us to differentiate the respondents according to their place of practice with a view to a possible comparison. In addition, the principle of self-assessment remains subjective, influenced by the desire to answer correctly. Finally, we could think that only practitioners interested in the subject have answered this questionnaire based on voluntary service. As the collection period coincided with the presence of coronavirus disease, many GPs remained inaccessible and some health centers were closed for a while. This notwithstanding the survey being declarative, with open and closed questions, was an advantage. In view of the number of responses, this was a common pathology with a marked interest in GPs.

\section{Conclusion}

Our study found that diagnostic criteria and acute treatment of migraine were well known by the majority of general practitioners, in contrast to preventive treatment. It is 
important to establish continuous training for GPs in order to optimize their knowledge, attitudes, and practices in the face of migraine.

$\begin{array}{ll}\text { Abbreviations } \\ \text { PCPs: } & \text { Primary care professionals } \\ \text { HCPs: } & \text { Healthcare providers } \\ \text { PHCP: } & \text { Primary healthcare providers } \\ \text { CM: } & \text { Chronic migraine } \\ \text { ICHD-3: } & \text { International Classification of Headache } \\ & \text { Disorders } \\ \text { NSAIDs: } & \text { Nonsteroidal anti-inflammatory drugs } \\ \text { IHS: } & \text { International Headache Society } \\ \text { VAS: } & \text { Visual analog scale } \\ \text { GPs: } & \text { General practitioners. }\end{array}$

\section{Data Availability}

The data used to support the findings of this study are available from the corresponding author upon request.

\section{Ethical Approval}

The authors had the authorization of Ethical Board Committee of Medical School of Saint Thomas D'Acquin University and authorization of data collection from Center Regional Direction of Heath before the survey. All applicable international, national, and/or institutional guidelines for the care and use of animals were followed. The privacy of the patients and the confidentiality of the data collected have been complied with.

\section{Consent}

All the participants had given their informed consent before the survey.

\section{Disclosure}

This article has not been published elsewhere (except for abstracts of conference materials).

\section{Conflicts of Interest}

The authors declare that they have no conflicts of interest.

\section{Authors' Contributions}

Dabilgou AA, Dravé A, Kyelem JMA, and Zoma A contributed to the review of the literature, writing, and editing of this manuscript. Napon C, Millogo A, and Kaboré J contributed to the concept and the design of this study. All authors have read and approved the final manuscript for publication.

\section{Acknowledgments}

The authors would like to thank all the participants of this study.

\section{References}

[1] P. J. Goadsby, R. B. Lipton, and M. D. Ferrari, "Migrainecurrent understanding and treatment," New England Journal of Medicine, vol. 346, no. 4, pp. 257-270, 2002.

[2] J. Olesen, "Headache Classification Committee of the International Headache Society (IHS). The international classification of headache disorders, 3rd edn (beta version)," Cephalalgia, vol. 33, pp. 629-808, 2013.

[3] R. C. Burch, S. Loder, E. Loder, and T. A. Smitherman, "The prevalence and burden of migraine and severe headache in the United States: updated statistics from government health surveillance studies," Headache: The Journal of Head and Face Pain, vol. 55, no. 1, pp. 21-34, 2015.

[4] World Health Organization, Headache Disorders, World Health Organization, Geneva, Switzerland, 2016.

[5] F. G. Freitag, "The cycle of migraine: patients' quality of life during and between migraine attacks," Clinical Therapeutics, vol. 29, no. 5, pp. 939-949, 2007.

[6] R. B. Lipton, S. Diamond, M. Reed, M. L. Diamond, and W. F. Stewart, "Migraine diagnosis and treatment: results from the American migraine study II," Headache: The Journal of Head and Face Pain, vol. 41, no. 7, pp. 638-645, 2001.

[7] E. A. MacGregor, J. Brandes, and A. Eikermann, "Migraine prevalence and treatment patterns: the global migraine and zolmitriptan evaluation survey," Headache: The Journal of Head and Face Pain, vol. 43, no. 1, pp. 19-26, 2003.

[8] T. R. Smith, R. A. Nicholson, and J. W. Banks, "Migraine education improves quality of life in a primary care setting," Headache: The Journal of Head and Face Pain, vol. 50, no. 4, pp. 600-612, 2010.

[9] A. F. H. Smelt, J. W. Blom, F. Dekker et al., "A proactive approach to migraine in primary care: a pragmatic randomized controlled trial," Canadian Medical Association Journal, vol. 184, no. 4, pp. E224-E231, 2012.

[10] N. Karli, M. Zarifoglu, S. Erer, K. Pala, and N. Akis, "The impact of education on the diagnostic accuracy of tensiontype headache and migraine: a prospective study," Cephalalgia, vol. 27, no. 1, pp. 41-45, 2007.

[11] M. A. Kaci, M. Haddad, B. Kouassi, and H. Ouhabi, "Consensus formalisé: recommandations de pratiques cliniques pour la prise en charge de la migraine du patient adulte africain," Pan African Medical Journal, vol. 81, no. 24, Article ID 12, 2016.

[12] T. J. Steiner, G. L. Birbeck, R. H. Jensen, Z. Katsarava, L. J. Stovner, and P. Martelletti, "Headache disorders are third cause of disability worldwide," The Journal of Headache and Pain, vol. 16, Article ID 58, 2015.

[13] M. E. Bigal and R. B. Lipton, "The epidemiology, burden, and comorbidities of migraine," Neurologic Clinics, vol. 27, no. 2, pp. 321-334, 2009.

[14] Y. W. Woldeamanuel, A. P. Andreou, and R. P. Cowan, "Prevalence of migraine headache and its weight on neurological burden in Africa: a 43-year systematic review and meta-analysis of community-based studies," Journal of the Neurological Sciences, vol. 342, no. 1-2, pp. 1-15, 2014.

[15] R. Ndejjo, G. Musinguzi, X. Yu et al., "Occupational health hazards among healthcare workers in Kampala, Uganda," Journal of Environmental and Public Health, vol. 2015, Article ID 913741, 9 pages, 2015.

[16] Y. W. Lin, Y. W. Chang, and C. C. Tsai, "Job strain and healthrelated quality of life of hospital employees: case of a medical center in Taichung," Taiwan Journal of Public Health, vol. 23, pp. 108-120, 2004. 
[17] M. Gultekin, E. Balci, S. Ismailogullari et al., "Awareness of migraine among primary care physicians in Turkey: a regional study," Noro Psikiyatri Arsivi, vol. 55, 2016.

[18] J. M. Martínez Eizaguirre, S. Calero Muñoz, M. L. García Fernández, S. Tranche Iparraguirre, J. Castillo Obeso, and I. PIrazusta, "Attitudes of Spanish primary care doctors to migraine,” Atención Primaria, vol. 38, pp. 33-38, 2006.

[19] S. Evers, N. Brockmann, and I. W. Husstedt, "Prevalence of migraine in headache specialists and Neurologists," Cephalalgia, vol. 27, Article ID 665, 2007.

[20] A. Malik, S. Awan, S. Aziz, F. Ahmed, and M. Wasay, "Awareness and approach to headache: a survey of family physicians in Pakistan," Pakistan Journal of Neurological Sciences, vol. 13, no. 4, pp. 5-10, 2018.

[21] R. Thomas, A. Cook, G. Main, T. Taylor, E. G. Caruana, and R. Swingler, "Primary care access to computed tomography for chronic headache," British Journal of General Practice, vol. 60, no. 575, pp. 426-430, 2010.

[22] G. R. Couchman, S. N. Forjuoh, M. H. Rajab, C. D. Phillips, and J. Yu, "Nonclinical factors associated with primary care physicians' ordering patterns of magnetic resonance imaging/ computed tomography for headache1," Academic Radiology, vol. 11, no. 7, pp. 735-740, 2004.

[23] M. Lanteri-Minet, D. Valade, G. Geraud, C. Lucas, and A. Donnet, "Revised French guidelines for the diagnosis and management of migraine in adults and children," The Journal of Headache and Pain, vol. 15, no. 1, Article ID 2, 2014.

[24] A. Ducros, S. Romatet, T. Saint Marc, and B. Allaf, "Use of antimigraine treatments by general practitioners," Headache: The Journal of Head and Face Pain, vol. 51, no. 7, pp. 11221131, 2011.

[25] M. Lantéri-Minet, H. Alchaar, G. Besson et al., "Pharmaco-epidemiological study on the prophylactic treatment of migraine. National inquiry on attitude to prescription practices by primary care physicians and neurologists in France," Revue Neurologique, vol. 156, no. 12, pp. 1106-1112, 2000.

[26] S. D. Silberstein, R. B. Lipton, D. W. Dodick et al., "Efficacy and safety of topiramate for the treatment of chronic migraine: a randomized, double-blind, placebo-controlled trial," Headache: The Journal of Head and Face Pain, vol. 47, no. 2, pp. 170-180, 2007.

[27] M. Silvestrini, M. Bartolini, M. Coccia, R. Baruffaldi, R. Taffi, and L. Provinciali, "Topiramate in the treatment of chronic migraine," Cephalalgia, vol. 23, no. 8, pp. 820-824, 2003.

[28] H.-C. Diener, G. Bussone, J. V. Oene, M. Lahaye, S. Schwalen, and P. Goadsby, "Topiramate reduces headache days in chronic migraine: a randomized, double-blind, placebo-controlled study," Cephalalgia, vol. 27, no. 7, pp. 814-823, 2007.

[29] B. Edvardsson, "Atenolol in the prophylaxis of chronic migraine: a 3-month open-label study," SpringerPlus, vol. 2, Article ID 479, 2013.

[30] Japanese Society of Neurology and the Japanese Headache Society, "Clinical practice guideline for chronic Headache," 2013, https://www.jhsnet.org/english/guideline2013.pdf.

[31] R. P. Silva-Neto, K. J. Almeida, and S. N. Bernardino, "Analysis of the duration of migraine prophylaxis," Journal of Neurological Sciences, vol. 337, no. 1-2, pp. 38-41, 2014.

[32] C. N. Mehrotra, N. Kanodia, P. Shankar, S. Amod Kumar, and D. Rakesh Kumar, "Knowledge, attitude and practice of general physicians related to migraine," World Journal of Pharmaceutical Sciences, vol. 3, no. 12, pp. 2470-2471, 2015.
[33] C. Fearon, K. Moloney, A. Chalissery, D. Ferguson, P. Moloney, and J. Redmond, “Assessment and management of patients with headache referred to a general neurology consultation service," Neurology, vol. 92, no. 15 Supplement, pp. 10-14, 2019. 